\title{
IL-13 antibody improves lung function in asthma
}

Results from a Phase II clinical trial of lebrikizumab (N. Engl.J. Med. 3 Aug 2011; doi:10.1056/NEJMoa1106469), a humanized monoclonal antibody that targets interleukin-13 (IL-13), have shown that the biologic improves lung function in adults with uncontrolled asthma.

Girolamo Pelaia, professor of respiratory medicine at the University 'Magna Graecia' of Catanzaro, Italy, explains why even though therapies such as inhaled corticosteroids and $\beta_{2}$-adrenergic receptor agonists control asthma symptoms and exacerbations in a large proportion of patients, there is a need for additional therapies. "A minority (5-10\%) of the overall asthmatic population use the largest share of health-care services, including emergency visits, hospitalizations and additional drugs because of their recurrent exacerbations. Therefore, these patients with severe uncontrolled asthma could benefit from the development of new drugs, including novel molecules that interfere with different aspects of airway inflammation, such as cytokine blockers."
In the trial of 219 individuals, $250 \mathrm{mg}$ of lebrikizumab (or placebo) was administered subcutaneously once a month for 6 months. Patients were grouped according to their serum levels of the matricelluar protein periostin, which was used as a surrogate marker of IL-13 activity.

IL-13 is involved in airway inflammation and remodelling, both of which contribute to asthma. "In particular, IL-13 induces isotype switching of $B$ cells towards immunoglobulin $E$ synthesis, increases expression of immunoglobulin E receptors on mast cells and causes upregulation of vascular cell adhesion molecule 1 , which is implicated in the microvascular recruitment of eosinophils and basophils," explains Pelaia. "Moreover, IL-13 also causes mucous metaplasia and increases production of collagen and fibronectin, which are responsible for the thickening of bronchial walls due to subepithelial fibrosis." So inhibiting IL-13 could benefit patients with asthma. However, because IL-4 is also involved in many of these processes, Pelaia notes that targeting both of these cytokines might have a more complete effect.

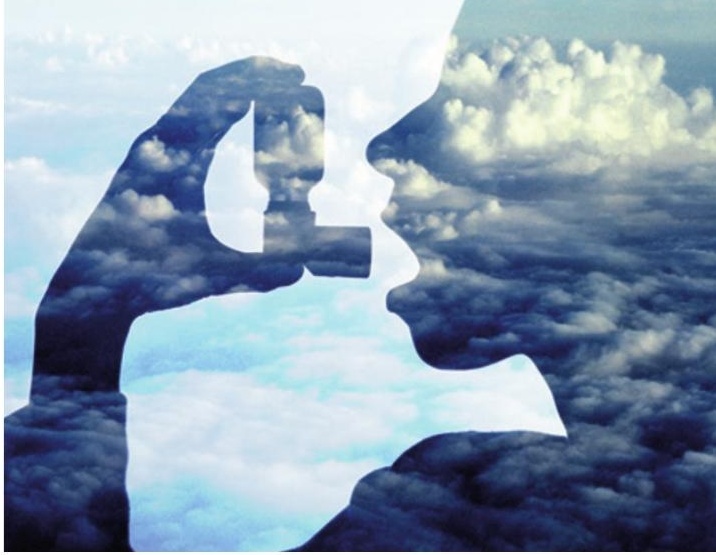

The primary efficacy outcome of the trial was the relative change in prebronchodilator-forced expiratory volume in 1 second $\left(F_{1} V_{1}\right)$ from baseline to week 12. Compared to the placebo group, the $\mathrm{FEV}_{1}$ of patients in the overall lebrikizumab group was $5.5 \%$ higher $(P=0.02)$; in the high-periostin subgroup the $\mathrm{FEV}_{1}$ was $8.2 \%$ higher $(P=0.03)$; and in the low-periostin subgroup the $\mathrm{FEV}_{1}$ was $1.6 \%$ higher $(P=0.61)$. There was a nonsignificant trend towards lower rates of severe exacerbations one of the secondary outcomes of the study - in the overall lebrikizumab group.

So this study showed that lebrikizumab improves lung function, and also indicates that a biomarker could be used to identify those patients most likely to respond to treatment. "The use of biomarkers - like periostin — to choose appropriate patients with asthma for specific treatments is an area of active research," says Jonathan Corren, of the Allergy Medical Clinic, Los Angeles, USA, who was an investigator on the trial. "Our hope is that reliable biomarkers will improve the initial choices of asthma therapy and optimize clinical outcomes, particularly in patients with more severe asthma." 